\title{
Caracterización de un modelo de diabetes tipo 2 en ratas Wistar hembra
}

\section{Characterization of a type 2 diabetes model in female Wistar rats}

\author{
M Consuelo Figueroa G, ${ }^{1}$ M.Sc, Ismael H Pérez $\mathrm{H}_{,}{ }^{2}$ M.Sc, Ricardo Mejía Z, ${ }^{2 *}$ Ph.D.
}

${ }^{1}$ Universidad Nacional Autónoma de México. Facultad de Medicina Veterinaria y Zootecnia. Circuito Interior S/N Av. Universidad No. 3000. Del Coyoacán. México, DF. ${ }^{2}$ Unidad de Biomedicina FESIUNAM. Av. de los Barrios No. 1, Los Reyes Iztacala, Tlalnepantla, México. *Correspondencia: rmejia@ campus.iztacala.unam.mx

Recibido: Noviembre de 2012; Aceptado: Marzo de 2013.

\section{RESUMEN}

Objetivo. Caracterizar un modelo de diabetes mellitus tipo 2 (DM2) en ratas hembra. Materiales y métodos. Se utilizaron 20 ratas Wistar hembra de 48 horas de edad; a 10 se les administró estreptozotocina (STZ), [135 mg/kg de peso corporal] en $50 \mu \mathrm{l}$ de amortiguador de citratos a pH de 4.5, vía intraperitoneal (inducidos) y a 10 se les administró $50 \mu \mathrm{l}$ del amortiguador (testigo). Resultados. Los animales inducidos presentaron glicemias de $7.8 \pm 2 \mathrm{mmol} / \mathrm{L}(52.9 \%$ más que el testigo; $p<0.005)$ y un peso de $334.4 \pm 12 \mathrm{~g}(51.6 \mathrm{~g}$ menor que el testigo; $\mathrm{p}<0.05)$ al final del estudio. La concentración de colesterol fue del doble en el grupo inducido. La concentración de triglicéridos no presentó diferencia entre grupos al primer mes de edad, pero al segundo fue $50 \%$ mayor en los animales inducidos $(p<0.05)$. La concentración de hemoglobina glicada fue de $4.3 \%$ en los testigos y de $5.9 \%$ en animales inducidos $(p<0.05)$. La curva de tolerancia mostró alteración en el grupo inducido con glicemias superiores a $7.4 \mathrm{mmol} / \mathrm{L}$ hasta los $240 \mathrm{~min}$. Conclusiones. De acuerdo con los parámetros de las pruebas comúnmente utilizadas para el diagnóstico y control de la diabetes en humanos, los valores obtenidos en el presente estudio convalidan el desarrollo de un modelo de DM2 en ratas hembras mediante el uso de STZ.

Palabras clave: Colesterol, hiperglicemia, rata (Fuente: DeCS).

\section{ABSTRACT}

Objective. Characterization of a type 2 diabetes mellitus (DM2) model in female rats. Materials and methods. 20, 48 hour-old wistar female rats were used; 10 were intraperitoneally injected with streptozotocin (STZ) [135 mg/kg body weight] in $50 \mu \mathrm{l}$ citrate buffer $\mathrm{pH} 4.5$ (induced group) and 10 were injected with $50 \mu \mathrm{l}$ of citrate buffer (control group). Results. The induced group had average glycemias of $7.8 \pm 2 \mathrm{mmol} / \mathrm{L}(52.9 \% ; \mathrm{p}<0.005)$ and a body weight of $334.4 \pm 12 \mathrm{~g}(51.6 \mathrm{~g}$ lower than the control group; $p<0.05$ ) at the end of the treatment. The cholesterol concentration was two-fold higher in the induced group. Triglyceride concentration was not different during the first month, but it 
was $50 \%$ higher on the second month for the induced group $(p<0.05)$. The concentration of glycated hemoglobin was $4.3 \%$ for the control group and $5.9 \%$ for the induced group $(p<0.05)$. The glucose tolerance curve was altered in the induced group with blood glucose concentrations in diabetic animals over $7.4 \mathrm{mmol} / \mathrm{L}$ until $240 \mathrm{~min}$. Conclusions. According to the parameters currently used for diagnostic and control of diabetes in humans, the results displayed in this study support the development of a DM2 model in female rats through the use of STZ.

Key words: Cholesterol, Hyperglycemia, rat (Source: DeCS).

\section{INTRODUCCIÓN}

La Organización Mundial de la Salud (OMS) calcula que en el mundo hay más de 250 millones de personas con diabetes, de las cuales el $90 \%$ de los pacientes son diagnosticados como Tipo 2; es probable que esta cifra aumente a más del doble en 2030 y se estima que un $30 \%$ de la población con DM2 no ha sido diagnosticada (1). La diabetes se clasifica en las siguientes categorías: Diabetes Mellitus Tipo 1 (DM1), producida por una deficiencia absoluta en la secreción de insulina; Diabetes Mellitus Tipo 2 (DM2), causada por una combinación de múltiples factores, como resistencia a la acción de la insulina y/o una inadecuada respuesta compensatoria del páncreas; diabetes gestacional (DMG), se presenta por alteraciones hormonales durante el embarazo y diabetes idiopática (transitoria) (2-3).

De acuerdo con la Asociación Americana de Diabetes (ADA, por sus siglas en inglés) y la OMS, los criterios para el diagnóstico de la diabetes mellitus son: a) la presencia de síntomas clásicos (polidipsia, polifagia, poliuria y pérdida de peso), con el hallazgo casual, sin considerar el tiempo pasado desde la última comida, de un nivel de glucosa en sangre (glucemia) por encima de $200 \mathrm{mg} / \mathrm{dl}(11.1 \mathrm{mmol} / \mathrm{L}) ;$ b) concentración de glucosa en ayunas superior a $126 \mathrm{mg} / \mathrm{dl}$ (7 $\mathrm{mmol} / \mathrm{L})$ y c) concentraciones de glucosa por encima de $200 \mathrm{mg} / \mathrm{dl}$ (11.1 mmol/L) en un análisis de dos horas posterior a una sobrecarga oral de glucosa de $75 \mathrm{~g}$ (test realizado según los criterios de la OMS). El hallazgo aislado de cualquiera de estos criterios no es suficiente para establecer el diagnóstico (2).

De igual manera se pueden evaluar algunos otros indicadores de alteraciones en el metabolismo de carbohidratos y lípidos que concuerdan con el desarrollo de la enfermedad como son: concentraciones sanguíneas alteradas de colesterol, triglicéridos, péptido $\mathrm{C}$, el índice de resistencia a la insulina (HOMA-IR; homeostatic model assessment) y hemoglobina glicada (3).

La fisiopatología de la DM2 es compleja e implica factores ambientales (el sedentarismo, la edad, bajo peso al nacer y dietas hipercalóricas que conducen a la obesidad) y genéticos. Existen tres alteraciones constantes en este padecimiento: resistencia a la acción de la insulina en los tejidos periféricos (músculo, tejido adiposo y especialmente hígado); secreción alterada de la insulina en respuesta al estímulo de glucosa y aumento en la producción de glucosa hepática (3). La resistencia periférica a la insulina se presenta con una fase preclínica (en estadio de tolerancia alterada a la glucosa), además una elevación moderada de la concentración de glucosa en sangre y elevadas concentraciones de colesterol y triglicéridos $(4,5)$. Con frecuencia este tipo de diabetes es diagnosticada varios años después de iniciada ya que en sus primeras etapas es casi asintomática (3).

El uso de los animales como modelos de experimentación en el estudio de la fisiopatología de la diabetes ha permitido entender algunas de sus causas y consecuencias, además de obtener avances en el tratamiento y control de este síndrome, aun cuando los signos y alteraciones no se reproducen con exactitud. En la literatura se han reportado modelos experimentales de diabetes en diferentes especies que van desde el perro, gato, conejo, cerdo, borrego, ratón hasta la rata $(4,6)$. En animales de laboratorio, específicamente en la rata, la diabetes se puede presentar de forma espontánea o inducida experimentalmente por distintos métodos (710). En la inducción experimental de diabetes se utilizan sustancias químicas que destruyen en forma selectiva las células $\beta$ del páncreas como son la alloxana (Alx) y la estreptozotocina (STZ). La inducción de la DM1 se realiza en animales adultos con dosis de $40 \mathrm{mg}$ de $\mathrm{Alx} / \mathrm{kg}$ de peso corporal (10) o de 50-60 mg de STZ/kg de peso $(8,11)$. Para el desarrollo de la DM2, se pueden utilizar neonatos de 1 a 5 días de vida y concentraciones de STZ entre 100 y 200 mg/kg de peso corporal $(9,12)$.

Existe abundante información acerca de las características y datos clínicos de la DM1 en ratas de laboratorio $(7-8,10)$; sin embargo, casi todos ellos han sido desarrollados en machos. 
Por otra parte, existe menos información de modelos experimentales en animales para la DM2 y prácticamente no se encuentran datos de ninguna de las dos categorías en hembras. En los modelos experimentales con animales se usa en forma convencional a los machos ya que el comportamiento de ciertos parámetros a lo largo del tiempo tiene menos fluctuaciones debido a que estos no presentan estados fisiológicos como el ciclo estral y el menstrual, la gestación y la lactancia, en los que cambios hormonales provocan ajustes en el metabolismo.

El objetivo de este trabajo fue caracterizar un modelo de diabetes mellitus tipo 2 en ratas Wistar hembras.

\section{MATERIAL Y MÉTODOS}

Animales de experimentación. Se utilizaron 10 ratas Wistar hembras de 9 semanas de edad, con un peso promedio de $250 \mathrm{~g}$, albergadas en forma individual en jaulas de polisulfonato con sistema de microaislamiento, con alimento y agua ad libitum y fotoperíodo de 12 horas luz y 12 obscuridad, obtenidas del Bioterio de la Facultad de Estudios Superiores Iztacala-UNAM. Se realizó frotis vaginal a los animales para diagnóstico de estro, aquellos que resultaron positivos se pusieron con machos sanos de la misma estirpe toda la noche para su apareo. Durante la mañana se realizó diagnóstico de apareo mediante frotis vaginal.

Tratamiento. Se seleccionaron ratas hembras de 48 a 72 horas de edad, obtenidas en pares (con lo que cada animal sometido a tratamiento tenía su control de la misma camada) en un sistema completamente aleatorio. A 10 ratas se les indujo diabetes tipo 2 mediante la administración vía intraperitoneal de $135 \mathrm{mg}$ de STZ/kg de peso corporal en $50 \mu \mathrm{l}$ de amortiguador de citratos a $\mathrm{pH}$ de 4.5 y 10 fueron utilizadas como grupo testigo con la administración de $50 \mu \mathrm{l}$ del mismo amortiguador. Los animales fueron mantenidos con la madre hasta el destete (cuatro semanas de edad) y posteriormente albergadas en grupos de 3 animales, en cajas de polisulfonato con microambiente y fotoperíodo de 12 horas luz, 12 horas oscuridad a $22^{\circ} \mathrm{C}$ en promedio, con alimento (2040 de Harlan ${ }^{\circledR}$ ) y agua ad líbitum. Se tomó como criterio de inclusión en el grupo tratado que los animales tuvieran una concentración de glucosa sanguínea menor a $200 \mathrm{mg} / \mathrm{dl}(11.11$ $\mathrm{mmol} / \mathrm{l}$ ) en ayuno de 8 horas y menor de $110 \mathrm{mg} /$ dl $(6.1 \mathrm{mmol} / \mathrm{L})$ en el grupo testigo. Los animales fueron mantenidos hasta las 16 semanas de edad, siguiendo los lineamientos de bioética marcados en la norma oficial mexicana (NOM
062-ZOO-1999) (13) y al término del estudio los animales fueron sacrificados con una sobredosis de pentobarbital sódico, vía intraperitoneal como lo marca la norma.

Cuantificación de glucosa. Se determinó glucosa (una vez por semana) a partir de la cuarta semana y hasta las 16 semanas de edad, con un equipo Accutrend ${ }^{\circledR}$ de Roche.

Cuantificación de triglicéridos y colesterol. Las cuantificaciones de colesterol y triglicéridos en sangre se realizaron una vez por mes a partir de la cuarta semana hasta la semana 16 , con un equipo Accutrend ${ }^{\circledR}$ de Roche.

Cuantificación de hemoglobina glicada. La cuantificación de hemoglobina glicada se realizó a las 16 semanas de edad con un equipo para medición instantánea $\mathrm{A} 1 \mathrm{CNow}+$ de Bayer $^{\circledR}$.

Curva de tolerancia a la glucosa (CTG). Se realizó a las 16 semanas de edad, mediante la administración oral (con sonda esofágica) de 3 $\mathrm{g}$ de dextrosa/kg de peso corporal en $3 \mathrm{ml}$ de agua; previa medición de los niveles basales de glucosa sanguínea. Posterior a la administración de la dextrosa, se realizaron mediciones de las concentraciones de glucosa sanguínea, cada 30 minutos, hasta 240 minutos.

Cuantificación de peso corporal. La medición del peso corporal se realizó (una vez por semana) a partir de la cuarta semana y hasta las 16 semanas de edad, con una báscula granataria.

Para la realización de las pruebas se mantuvo a los animales en ayuno durante 8 horas y las mediciones se realizaron a las 18:00 horas.

Análisis estadístico. Los resultados obtenidos fueron analizados por medio de un análisis de varianza (ANOVA) de una vía y prueba de Tukey para identificar diferencias entre tratamientos $(p<0.05)$, usando el software JMP (Versión 7.0) (14).

\section{RESULTADOS}

Los animales inducidos a DM2 en este estudio presentaron glicemias por arriba de los parámetros marcados como normales en el $75 \%$ de los tiempos evaluados, mientras que los animales del grupo testigo, el $100 \%$ de las ocasiones se mantuvieron por debajo del límite máximo considerado como normal (6.1 $\mathrm{mmol} / \mathrm{L})$, (Figura 1). La concentración promedio de glucosa en el grupo testigo fue de $5.1 \pm 0.88$ $\mathrm{mmol} / \mathrm{L}$, mientras que los animales inducidos 
a DM2 tuvieron concentraciones promedio de $7.8 \pm 2 \mathrm{mmol} / \mathrm{L}$, observándose diferencia estadísticamente significativa entre tratamientos $(p<0.05)$ en el período de 80 a 105 días de edad.

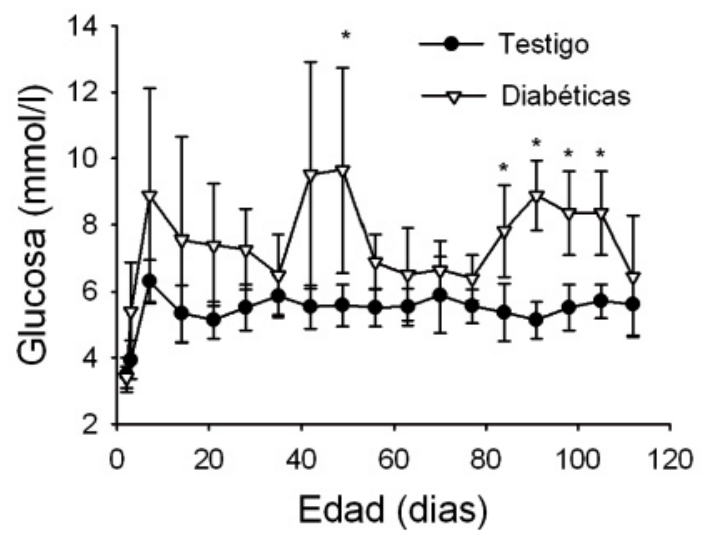

Figura 1. Comportamiento Tiempo/tratamiento de la concentración de Glucosa ( $\mathrm{mmol} / \mathrm{l})$ en ratas inducidas a diabetes tipo 2 (STZ $135 \mathrm{mg} /$ $\mathrm{kg}$ ) y su control no inducido. * $\mathrm{p}<0.05$.

En la figura 2 se presentan las curvas de crecimiento del grupo inducido a diabetes y su testigo. Se puede observar que el peso de las ratas testigo (386 $\pm 18 \mathrm{~g})$ es mayor que el de las ratas inducidas a DM2 (334.4 $\pm 12 \mathrm{~g})$ a las 16 semanas de edad. La ganancia diaria de peso de los animales diabéticos durante el estudio es menor que la del grupo testigo, encontrando que en el $60 \%$ de los momentos estudiados existe diferencia estadísticamente significativa entre tratamientos $(p<0.05)$.

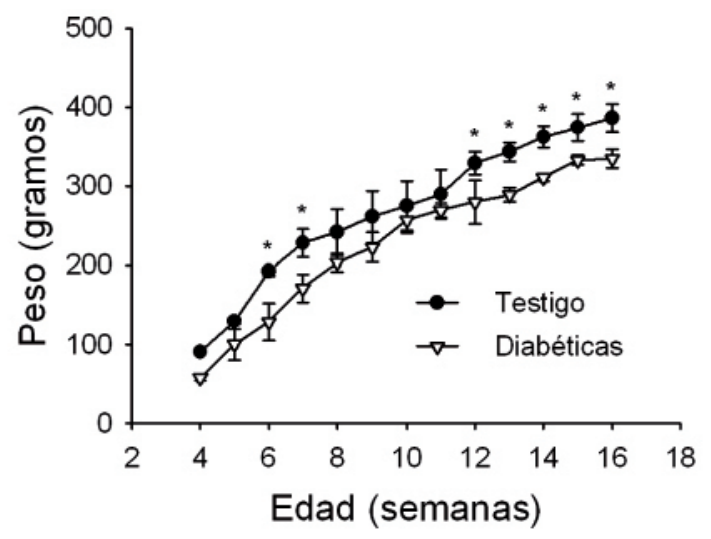

Figura 2. Curva de crecimiento en ratas inducidas a diabetes tipo 2 y su control. $* \mathrm{p}<0.05$.
La concentración de colesterol entre el primer y cuarto mes de edad en los animales del grupo testigo (entre 85 y $98 \mathrm{mg} / \mathrm{dl}$ ) es $50 \%$ menor que en los inducidos a diabetes (entre 169 y 178 $\mathrm{mg} / \mathrm{dl}$ ), existiendo diferencia estadísticamente significativa entre tratamientos $(p<0.05)$. (Figura 3).

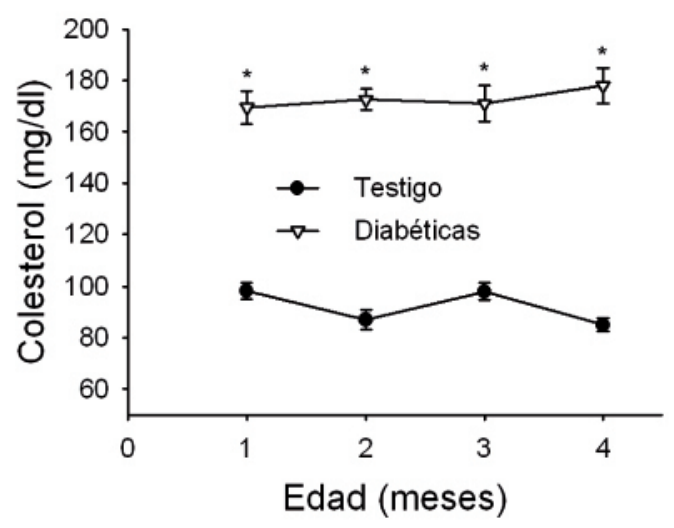

Figura 3. Concentración de Colesterol en ratas inducidas a diabetes tipo 2 y su control. $* \mathrm{p}<0.05$.

En la figura 4 se presentan los resultados de la concentración de triglicéridos. Se puede observar que no existe diferencia estadísticamente significativa entre grupos en el primer mes de vida $(83.2 \pm 8.2 \mathrm{mg} / \mathrm{dl}$ en testigos vs. $90.3 \pm 7.7$ $\mathrm{mg} / \mathrm{dl}$ en inducidas). A partir del segundo mes de edad existe una tendencia a incrementar la concentración en ambos grupos, donde siempre es significativamente $(p<0.05)$ mayor la concentración de triglicéridos en las ratas inducidas $(123.8 \pm 12.1,125.8 \pm 6.5$ y $235 \pm 17$ $\mathrm{mg} / \mathrm{dl}$ a 2 , 3 y 4 meses respectivamente en el grupo testigo y de $187 \pm 18.6,199.8 \pm 25$ y

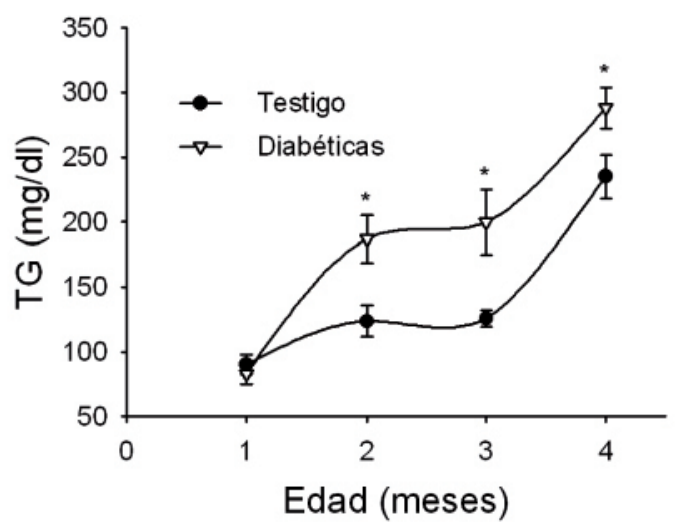

Figura 4. Concentración de Triglicéridos en ratas inducidas a diabetes tipo 2 y su control. $* \mathrm{p}<0.05$. 
$288 \pm 15.8 \mathrm{mg} / \mathrm{dl}$ a 2,3 y 4, meses en el grupo inducido).

En el presente estudio se observaron concentraciones de hemoglobina glicada de $4.3 \%$ en los animales testigo y de $5.9 \%$ en animales inducidos a diabetes Tipo 2, con diferencia estadísticamente significativa $(p<0.05)$ (Figura 5).

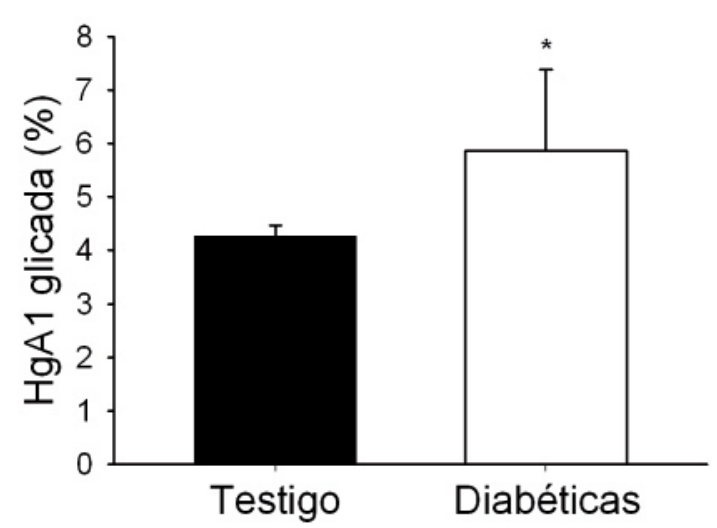

Figura 5. Concentración de Hemoglobina glicada en ratas inducidas a diabetes tipo 2 y su control. * $\mathrm{p}<0.05$.

En la curva de tolerancia a la glucosa (CTG), el grupo testigo muestra una concentración de 5.1 $\mathrm{mmol} / \mathrm{L}$ al inicio de la prueba, llega a valores máximos ( $9.7 \mathrm{mmol} / \mathrm{L})$ a la media hora después de la descarga oral de dextrosa y regresa a niveles basales a los 60 minutos. Los animales inducidos a diabetes alcanzaron concentraciones pico de $19.3 \mathrm{mmol} / \mathrm{L}$ a los 60 minutos y un retorno a $7.4 \mathrm{mmol} / \mathrm{L}$ hasta los 240 minutos, sin alcanzar el valor inicial de $6.6 \mathrm{mmol} / \mathrm{L}$, como se observa en la figura 6.

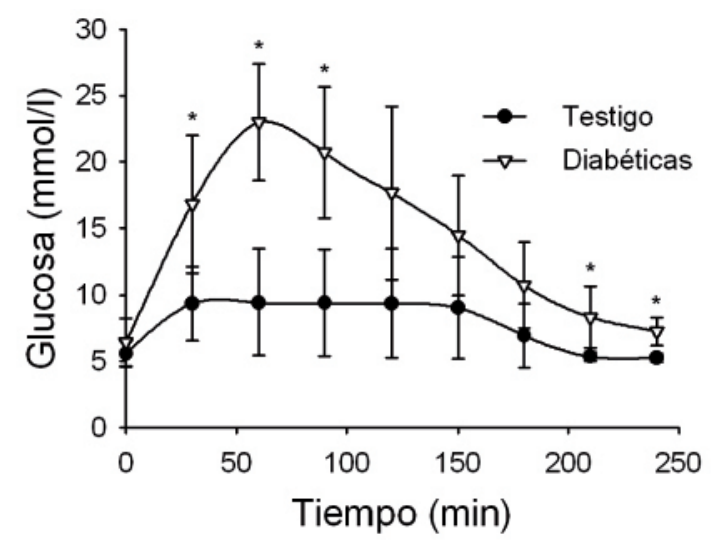

Figura 6. Curva de tolerancia a la glucosa en ratas inducidas a diabetes tipo 2 y su control. $* \mathrm{p}<0.05$.

\section{DISCUSIÓN}

El comportamiento aparentemente errático de la concentración de glucosa sanguínea a través del tiempo en las ratas inducidas a DM2 con STZ durante el estudio es una respuesta lógica a las necesidades de readaptación metabólica del individuo y los sistemas de contrarregulación hormonal de la glucosa (15). En pacientes humanos con diabetes tipo 2 no controlada, son comunes los hallazgos de glicemias que pueden ir de valores tan bajos como $2.77 \mathrm{mmol} / \mathrm{L}$ a valores que sobrepasan los $22.2 \mathrm{mmol} / \mathrm{L}$. Esta condición es una de las principales características que indican una mala utilización y/o síntesis de la insulina (16-18), aunque es necesaria la confirmación a través de pruebas como HOMA-IR, concentración de insulina y péptido $C$ en plasma. Se conoce que la STZ actúa específicamente sobre las células $\beta$ del páncreas utilizando al transportador de glucosa (GLUT 2) para entrar a la célula, causando daño y muerte de esta por la polirribosilación del DNA y la generación de radicales a través de la vía xantina-oxidasa (10).

En este trabajo se utilizó una concentración de STZ de $135 \mathrm{mg} / \mathrm{kg}$ de peso corporal, suficiente para disminuir a casi su totalidad las células $\beta-$ pancreáticas. Sin embargo, en animales neonatos el páncreas tiene la capacidad de regenerar $\mathrm{y} / \mathrm{o}$ diferenciar parcialmente la población celular, lo que resulta en un animal adulto con hiperglucemias moderadas. Lo anterior ha sido demostrado por nuestro grupo de trabajo mediante la utilización de técnicas de inmunohistoquímica que evidencian la disminución de la población de células $\beta$, estos datos fueron corroborados por la baja concentración de insulina en sangre y episodios de hiperglicemia característicos de DM2 (19). Probablemente la dispersión de los datos de las concentraciones de glucosa sanguínea observados en la figura 1 , se relaciona con los procesos de homeorresis utilizados para mantener la glicemia en condiciones estables. Sin embargo, la glucotoxicidad y la lipotoxicidad permanentes continúan causando daño lo que lleva nuevamente al aumento de la glucemia.

La DM2 se diagnostica por la presencia de los signos y síntomas clásicos, glucemia elevada en ayunas y/o curva de tolerancia a la glucosa alterada. En pacientes con sintomatología de diabetes la ADA especifica que sólo se necesita una glicemia mayor a $6.1 \mathrm{mmol} / \mathrm{L}$ en ayuno de 8 horas, o de $11.1 \mathrm{mmol} / \mathrm{L}$ en forma aleatoria para su confirmación. La OMS da como positivos a aquellos pacientes que en forma repetida dan como resultado una concentración de glucosa sanguínea mayor a $6.1 \mathrm{mmol} / \mathrm{L}$ en ayuno de 8 horas $(2,20)$. 
La concentración de glucosa sanguínea en los animales con DM2 es $52.9 \%$ más alta que la reportada en la literatura $(5.27 \mathrm{mmol} / \mathrm{L})(2)$. Los valores promedio en ratas con DM2 reportados por diversos autores van de 6.6 a $19.4 \mathrm{mmol} / \mathrm{L}$, dependiendo del manejo nutricional y estado fisiológico de los animales. En humanos, una concentración de glucosa sanguínea mayor a $6.1 \mathrm{mmol} / \mathrm{L}$ es un indicador de la disminución de la sensibilidad del receptor de insulina y se reportan como pacientes pre-diabéticos, a los cuales hay que realizar una prueba de tolerancia a la glucosa para confirmar o descartar DM2. La administración de STZ a una dosis de $135 \mathrm{mg} / \mathrm{kg}$ de peso corporal vía intraperitoneal en ratas Wistar hembras a los 2 días de edad, genera glicemias entre 5.4 y $10.6 \mathrm{mmol} / \mathrm{L}$, teniendo un promedio muestral a lo largo del tiempo de $7.8 \pm 1.45 \mathrm{mmol} / \mathrm{L}$, la dispersión de los valores obtenidos es consistente con el desarrollo de un modelo de Diabetes de Tipo 2 (Figura 1).

Uno de los parámetros afectados en pacientes diabéticos es el peso corporal. La literatura señala que los pacientes, previo al desarrollo de la diabetes Tipo 2, cursan con síndrome metabólico caracterizado por sobrepeso, intolerancia a la glucosa y alteración del metabolismo de los lípidos; sin embargo, ya establecida la enfermedad los pacientes presentan pérdida progresiva de peso corporal (21). En el presente estudio se puede observar que el efecto de la diabetes sobre esta variable (pobre ganancia de peso) concuerda con los datos reportados en la literatura donde se han realizado estudios para evaluar el efecto de ciertos fármacos sobre la concentración de la glucosa en sangre y ganancia de peso corporal en ratas (Figura 2 ). Hay que considerar que en este estudio los animales utilizados se encuentran en la etapa de desarrollo, definiéndose la pobre ganancia de peso como un estado de desnutrición celular, ocasionado por el ineficiente aporte de glucosa, teniendo como resultado el aumento en el catabolismo y un bajo anabolismo $(3,5)$.

La insulina, además de regular la utilización de la glucosa, tiene como función la regulación del metabolismo del tejido adiposo (almacenamiento, movilización y utilización de triglicéridos). Una de las alteraciones metabólicas observada en pacientes con diabetes Tipo 2 es el metabolismo de los lípidos, lo cual se ve reflejado en aumento de la concentración de triglicéridos y colesterol en sangre. Los datos reportados en la literatura para la concentración de estas moléculas en suero de ratas normales es de $70 \mathrm{mg} / \mathrm{dl}$ para colesterol (22) este valor es cercano al que se observó en los animales del grupo testigo (entre 85 y $98 \mathrm{mg} / \mathrm{dl}$ ) mientras que los animales inducidos a diabetes
Tipo 2 este valor se incrementa al doble (Figura 3 ), lo cual es similar a lo que ocurre en pacientes con DM2. La exposición crónica de células $\beta$ a altas concentraciones extracelulares de ácidos grasos produce la pérdida de la sensibilidad a la glucosa mostrando un patrón de hipersecreción de insulina a bajas concentraciones del azúcar y una incapacidad de adaptar la respuesta secretora a incrementos en la glucemia. La síntesis de insulina se ve severamente disminuida y se observa además una deposición exagerada de lípidos, cuya degradación se ve reducida incluso tras varios días en condiciones normoglucémicas, aumentando la posibilidad de daños por lipotoxicidad (18).

Por otro lado, es interesante el hecho de que al primer mes de tratamiento, la concentración de triglicéridos es la misma en los dos grupos, lo cual significa que aún no se ha generado daño en lo que al metabolismo de los triglicéridos se refiere. Sin embargo, para el segundo mes de tratamiento y en adelante, hay un claro aumento en ambos grupos, aunque este es mayor en el grupo inducido a diabetes (Figura 4). Este fenómeno puede estar relacionado con cambios en el medio ambiente, ciclos hormonales $\mathrm{y} / \mathrm{o}$ factores estresantes en ambos grupos; sin embargo el mayor incremento en el grupo inducido sugiere además alteración en la actividad y efectos de la insulina, entre los que se encuentran promover el almacenamiento de grasa e inhibir el catabolismo de ésta $(3,15)$. Los valores de triglicéridos en ratas reportados por diversos autores son variables y van de concentraciones de 80 a $160 \mathrm{mg} / \mathrm{dl}$ por lo que se tomó como valor de comparación el obtenido en este estudio.

La medición de la homoglobina glicada es una de las pruebas que se sugieren para el monitoreo del correcto control de los niveles de glucosa a través del tiempo, ya que es una reacción que se da de forma espontánea en un cierto porcentaje del total de esta proteína y es proporcional al tiempo de exposición y concentración de glucosa sanguínea. En ratas normales se ha observado que la concentración de la hemoglobina glicada es de $4.6 \%$, similar a la encontrada en el presente estudio. Para el grupo inducido se obtuvieron concentraciones de $37.2 \%$ por arriba del grupo control, similares a las reportadas en animales con DM1 $(5.3 \%)(16,23)$, (Figura $5)$. Un factor que modifica las concentraciones de hemoglobina glicada es el período de vida del eritrocito ( 45 a 50 días), en la rata existen cinco tipos de poblaciones de eritrocitos, el I, II y III presentes al nacimiento, son sustituidos por IV y $V$ entre los días 20-24 de vida del animal. La población IV desaparece el día 84, 
después de lo cual la población $V$ constituye la variante definitiva, por lo que la medición de la hemoglobina glicada en períodos tempranos de vida es un valor poco confiable. En este estudio se realizó en animales de corta edad y por un período de 16 semanas, por lo que es probable que el tiempo de exposición de la hemoglobina de los eritrocitos $V$ a las altas concentraciones de glucosa no fue suficiente con lo cual el porcentaje de glicación de esta proteína puede ser variable.

Los criterios utilizados para definir la condición de anormalidad en una CTG se basa en el nivel o pico alcanzado por la glicemia a los 60 minutos, y la falta de retorno al nivel normal dos horas después de la ingestión, siendo esto último lo más importante. Los datos encontrados en la literatura señalan que la concentración máxima de glucosa sanguínea a los treinta minutos en CTG en animales normales es de $7.32 \mathrm{mmol} / \mathrm{L}$, con un regreso al nivel basal $(4.9 \mathrm{mmol} / \mathrm{L})$ a los 120 minutos (24-25). En machos inducidos a DM2 se reportan concentraciones pico de 27.3 $\mathrm{mmol} / \mathrm{L}$ a los 30 minutos con un retorno a niveles iniciales de $12.1 \mathrm{mmol} / \mathrm{L}$ a los 180 minutos. En el presente estudio, tanto el grupo testigo como el diabético tuvieron un comportamiento similar al reportado en machos $(19,24,26)$. Los valores plasmáticos de insulina y glucosa en ayuno y posterior a la ingestión de una carga de carbohidratos, guardan relación mutua. Por esta razón la respuesta de estas moléculas a la CTG puede pronosticarse con base en los valores respectivos durante el ayuno, habiendo una mayor correlación con la insulina que con la glucosa (24).

El incremento agudo de insulina disminuye la concentración de glucosa plasmática, mientras que a largo plazo disminuye la acción de la hormona, alterando la unión de ésta a sus receptores de membrana. La elevada concentración de glucosa sanguínea a largo plazo disminuye la secreción de la hormona gluco-inducida, que es una de las manifestaciones del fenómeno de toxicosis por dicho carbohidrato. El resultado global de este sistema homeostático y sus circuitos es que las dos variables (concentración de insulina-glucosa sanguínea) varían siempre en proporción directa y mutua (salvo que disminuya la secreción de insulina) (26-28).

La diabetes es una enfermedad multifactorial y multisistémica, en la cual el desarrollo, incidencia y prevalencia de la misma está relacionada con factores inherentes a variables como edad, etapa fisiológica, genotipo y sexo, además de la influencia de factores ambientales $(3,5,27,29)$. Se requiere del desarrollo de modelos experimentales que reproduzcan la historia natural de la enfermedad en hembras en los diferentes estados fisiológicos.

En el presente estudio se desarrollaron las alteraciones fisiologías reconocidas en la diabetes tipo 2 de acuerdo a los criterios para el diagnóstico de ésta propuestos por la OMS y la ADA. Considerando los parámetros de las pruebas comúnmente utilizadas para el diagnóstico y control de la diabetes en humanos, los valores obtenidos son representativos del síndrome, sin embargo, se recomienda realizar algunas otras mediciones para la confirmación del diagnóstico como son mediciones de insulina, péptido $\mathrm{C}$ y el HOMA-IR.

\section{Agradecimientos}

A CONACYT por el otorgamiento de la beca Doctoral número 25340 a MCFG en el Posgrado FMVZ- UNAM. Al Biólogo Tomas Villamar Duque y al M en C Fernando Barrón, Bioterio de la FESIUNAM. Este trabajo fue financiado por el Proyecto PAPIIT IN228610-3 DGAPA-UNAM.

\section{REFERENCIAS}

1 Feudtner C. Diabetes: la paradoja de la tecnología moderna. Boletín de la Organización Mundial de la Salud 2011; 89:90-91. doi:10.2471/BLT.11.040211. URL Disponible En: http://www.who.int/bulletin/ volumes/89/2/11-040211.pdf

2 American Diabetes Association. Diagnosis and Classification of Diabetes Mellitus. Diabetes Care 2011; 34(SUPPLEMENT 1), S62-S69. DOI: $10.2337 / \mathrm{dc} 11-S 062$.
3 Stumvoll M, Goldstein BJ, Haeften TW. Type 2 diabetes: principles of pathogenesis and therapy. The Lancet 2005; 365(9467):1333-1346.

4 Claypool MD, Entes LJ, Experimental rodent models of type 2 diabetes: a review. MFECP 2009; 31(4):249-261. 
5 Muoio DM, Newgard CB. Molecular and metabolic mechanisms of insulin resistance and $\beta$-cell failure in type 2 diabetes. Nat Rev Mol Cell Biol 2008; 9:193-205.

6 Entes LJ, Claypool MD, Pinkett JG, Gadbois tM, Reaven GM. A new rat model of type 2 diabetes: the fat-fed, streptozotocin-treated rat. Met Clin and Exp 2000; 49(11):1390-1394.

7 Andrés S, Fleitas EAS, Simón CR, Almeida G, Quintela PAM, Alfonso M. Modelo experimental de diabetes en conejos. Rev Cubana Angiol y Cir Vasc 2000; 1(1):10-15.

8 Fernández SG, Jardón DA, Figueroa HJL. Modelos experimentales utilizados para el estudio e investigación de la diabetes mellitus. $2^{\circ}$ Congreso Nacional de Química Médica. Querétaro, México; 2006.

9 González E. Diabetes mellitius experimental: etiología de las malformaciones congénitas en descendentes de ratas diabéticas. Rev Cubana Endocrinol 2002; 13(1):3-63.

10 Szkudelski T. The Mechanism of alloxan and streptozotocin action in B cells of rat páncreas. Physiol Res 2001; 50:536-544.

11 Meszaros K, Reaven GM. A new rat model of type 2 diabetes: the fat-fed, streptozotocintreated rat. Met Clin and Exp 2000; 49(11): 1390-1394.

12 Hugues HB, Rodríguez GJC. Animales de laboratorio en la endocrinología: biomodelos de la diabetes mellitus tipo 1 . Rev Cubana Endocrinol 2001; 12(3):168-177.

13 NOM-062-ZOO-1999. Diario Oficial. Especificaciones técnicas para el Cuidado y Uso de los Animales de Laboratorio. México: Norma Oficial Mexicana; 2001.

14 JMP. Statistical Discovery Software. V. 7.0. Cary, USA: SAS Institute Inc; 2007.

15 Fanelli C, Pampanelli S, Porcellati F, Bartocci L, Scionti L, Rossetti P, Bolli G. Rate of fall of blood glucose and physiological responses of counterregulatory hormones, clinical symptoms and cognitive function to hypoglycaemia in Type I diabetes mellitus in the postprandial state. Diabetologia 2003; 46(Issue 1):53-64.
16 Carvajal SG, Zamudio CP, Carvajal JME, Juárez CE. Prevención de los daños producidos por la diabetes mellitus y la senescencia. Gac Méd Méx 2007; 143(1)51-59.

17 Petersen KF, Dufour S, Befroy D, Garcia R, Shulman GI. Impaired mitochondrial activity in the insulin-resistant offspring of patients with type 2 diabetes. N Engl J Med 2004; 350(7):664-671.

18 Zhang $Y$, Li X, Zou D, Liu W, Yang J, Zhu $\mathrm{N}$, Huo L, Wang $M$, Hong J, Wu P, Ren G, Ning G. Treatment of type 2 diabetes and dyslipidemia with the natural plant alkaloid berberine. JCEM 2008; 93(7):2559-2565.

19 Orozco-Tapia RI. Efecto de los ácidos grasos omega-3 (FA $\omega-3$ ) y linoleico conjugado (CLA) en la diabetes mellitus 2 (DM2) en ratas wistar. [Tesis]. México. UNAM. Facultad de Estudios Superiores Iztacala. 2008.

20 Aronoff SL, Berkowitz K, Shreiner B, Want L. Glucose metabolism and regulation: Beyond insulin and glucagon. Diabetes Spectrum 2004; 17(3):183-190.

21 Druker DJ. The role of gut hormones in glucose homeostasis. J Clin Invest 2007; 117(1):24-32.

22 Kadowaki T, Yamauchi T, Kubota N, Hara K, Ueki K, Tobe K. Adiponectin and adiponectin receptors in insulin resistance, diabetes, and the metabolic syndrome. J Clin Invest 2006; 116(7): 1784-1792.

23 Rorsman P. Review: Insulin secretion: function and therapy of pancreatic beta cells in diabetes. The British Journal of Diabetes \& Vascular Disease 2005; 5:187-191.

24 Bhandari U, Jain N, Pillai KK. Further studies on antioxidant potential and protection of pancreatic $\beta$-Cells by embelia ribes in experimental diabetes. Experimental Diabetes Research. Indian J Pharmacol 2008; 40(5):215-220.

25 Fernandez-Twinn, Wayman A, Ekizoglou S, Martin MS, Hales CN, Ozanne SE. Maternal protein restriction leads to hyperinsulinemia and reduced insulin-signaling protein expression in 21-mo-old female rat offspring. Am J Physiol Regul Integr Comp Physiol 2005; 288:R368-R373. 
26 Redd MG, Meszaros K, Roche E. Type 2 diabetes: gluco-lipo-toxicity and B-cell dysfunction. Ars Pharmaceutica 2003; 44(4):313-332.

27 Strader AD, Clausen TR, Goodin SZ, Wendt D. Ileal interposition improves glucose Tolerance in low dose streptozotocin-treated diabetic and euglycemic rats. Obes Surg 2009; 19(1):96-104.
28 Parra GL, Reyes Téllez GJ, Briones BC. La desnutrición y sus consecuencias sobre el metabolismo intermedio. Rev Fac Med UNAM. 2003; 46(1):32-36.

29 Maureen I. Harris. "Definición y clasificación de la diabetes mellitus y criterios actuales para el diagnóstico". En LeRoith D, Taylor, S, Olefsky, J. "Diabetes Mellitus, Texto Básico y Clínico". México: Ed. McGraw-Hill Interamericana; 2003. 